\title{
A COMPUTATIONAL AND EXPERIMENTAL INVESTIGATION OF A DELTA WING WITH VERTICAL TAILS
}

\author{
Sherrie L. Krist ${ }^{\star}$ and Anthony E. Washburn* \\ ViGYAN, Inc. \\ Hampton, Virginia \\ Kenneth D. Visser ${ }^{\dagger}$ \\ National Research Council \\ Hampton, Virginia
}

\begin{abstract}
The flow over an aspect ratio 1 delta wing with twin vertical tails is studied in a combined computational and experimental investigation. This research is conducted in an effort to understand the vortex and fin interaction process. The computational algorithm used solves both the thin-layer Navier-Stokes and the inviscid Euler equations and utilizes a chimera grid-overlapping technique. The results are compared with data obtained from a detailed experimental investigation. The laminar case presented is for an angle of attack of $20^{\circ}$ and a Reynolds number of 500,000 . Good agreement is observed for the physics of the flow field, as evidenced by comparisons of computational pressure contours with experimental flow-visualization images, as well as by comparisons of vortex-core trajectories. While comparisons of the vorticity magnitudes indicate that the computations underpredict the magnitude in the wing primary-vortex-core region, grid embedding improves the computational prediction.

\section{Nomenclature}

$\begin{array}{ll}A R & \text { aspect ratio } \\ a & \text { speed of sound } \\ e & \text { total energy per unit volume } \\ \mathbf{F}, \mathbf{G}, \mathbf{H} & \text { inviscid fluxes } \\ \mathbf{H}_{v} & \text { viscous flux } \\ J & \text { transformation Jacobian, } \\ & J=\partial(\xi, \eta, \zeta) / \partial(x, y, z)\end{array}$

$L \quad$ reference length, taken as wing root chord

$M \quad$ Mach number, $M=|\widetilde{\mathbf{V}}| / \widetilde{a}$

$p \quad$ static pressure

$p_{0} \quad$ stagnation pressure

$\dot{q} \quad$ heat flux terms

\footnotetext{
Research Engineer, Member AIAA

$\dagger$ National Research Council Associate, Member AIAA
}

This paper is declared a work of the U.S. Government and

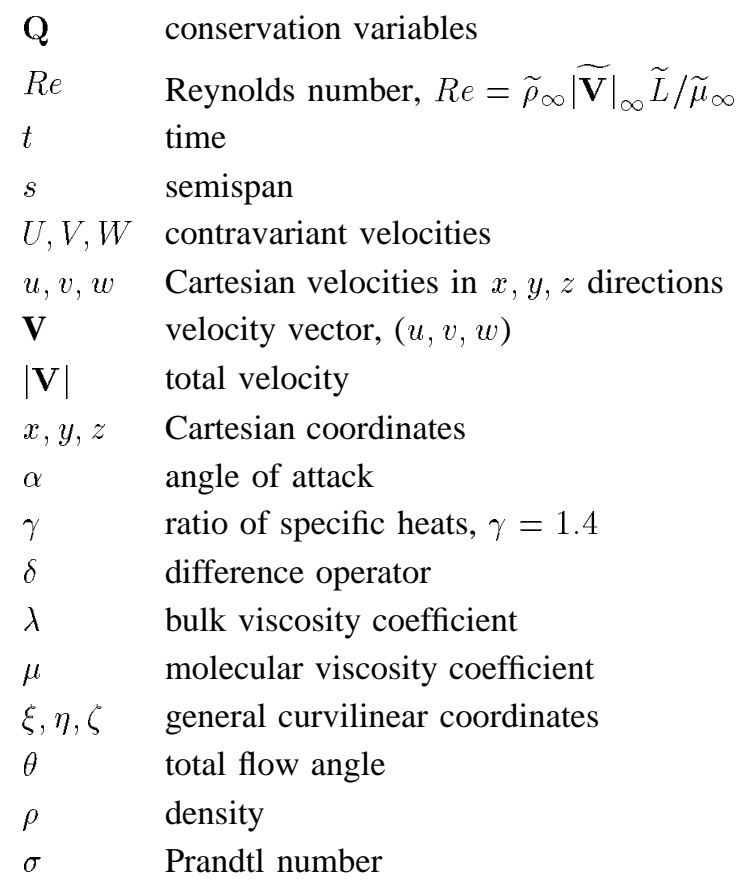
is not subject to copyright protection in the United States. 


$$
\begin{array}{ll}
\tau & \text { shear stress tensor } \\
\Omega & \text { vorticity vector, }\left(\Omega_{\mathrm{x}}, \Omega_{\mathrm{y}}, \Omega_{\mathrm{z}}\right)
\end{array}
$$

Subscripts:

$x, y, z \quad$ denotes differentiation with respect to

$x, y, z$

$\infty \quad$ denotes free-stream conditions

Superscripts:
$\sim \quad \begin{aligned} & \text { denotes quantities in generalized } \\ & \text { coordinates } \\ & \text { denotes dimensional value }\end{aligned}$

\section{Introduction}

Modern fighter aircraft have been designed for extreme maneuverability at high angles of attack. To achieve the required level of agility, highly swept planform surfaces have often been incorporated into the aircraft design to take advantage of the leading-edge vortices that are generated. Some of today's fighter aircraft, such as the F-18, have been designed with twin vertical tails located aft of the highly swept wings, in the path of the vortical flow field. At sufficiently high angles of attack, vortex breakdown can occur, which can lead to severe buffeting and potential structural fatigue in the tails.

Because the F-18 has encountered such problems, considerable effort has recently been directed toward understanding the physics of the vortex and tail interaction. Numerous experimental studies of this process have been conducted with the F-18. ${ }^{1,2,3}$ Rizk et al. ${ }^{4,5}$ computationally modeled the F-18 to study the effects of the wing vortices on the vertical tails. For the current investigation, an aspect ratio 1 delta wing with vertical tails has been modeled computationally for comparison with results from a detailed experimental investigation. ${ }^{6}$ This simplified geometry has been chosen to focus on only the vortex and tail interaction process; other features of the full-scale aircraft have been ignored in this analysis.

Cooperative studies with the Navier-Stokes code "CFL3D" for the computational investigation and NASA Langley's Basic Aerodynamic Research Tunnel for the experimental work have already examined the capability of CFL3D to accurately capture the delta-wing flow-field properties. In the previous studies, CFL3D correctly predicted the size and shape of the wing primary vortex, but revealed a deficiency in primary-vortex-core strength when compared with the experimental results. ${ }^{7}$ A subsequent study utilized a grid-embedding technique to refine the mesh in the primary-vortex region, which resulted in improved agreement with the experimental data. ${ }^{8}$ As an extension of the previous efforts, the current computational research investigates the flow field over an aspect ratio 1 delta wing with vertical tails.

For the computational study, the chimera, or gridoverlapping, technique developed by Benek et al. ${ }^{9}$ has been employed. The chimera method has been utilized for a variety of applications that include transonic wall interference, ${ }^{10}$ F-18 flow-field calculations, ${ }^{11}$ and the Space Shuttle launch vehicle. ${ }^{12}$ With the chimera technique, grids for individual components of a configuration can be generated independently of one another. This feature is particularly desirable for the present application because delta-wing meshes generated for previous applications can be utilized. An independently generated tail grid can then be inserted into the domain of the delta-wing grid. Communication between the grids is achieved through interpolation stencils.

The aspect ratio 1 ( $76^{\circ}$ sweep) delta-wing model, which is used both computationally and experimentally, has a sharp leading edge, a flat upper surface, and a triangular cross section. The wing is thin; a maximum thickness of 2.1 percent of the root chord occurs at $\widetilde{x} / \widetilde{L}=0.9$. The tails are located behind the wing at $\widetilde{y} / \widetilde{s}=0.78$ and $\tilde{x} / \widetilde{L}=1.06$ (referenced from the apex of the wing to the tail leading edge). The tails are oriented normal to the wing upper surface and have a leading-edge sweep of $62.5^{\circ}$ and a taper ratio of 0.23 . The test configuration selected for the present study was based on a previous parametric study conducted by Washburn et al. ${ }^{6}$ This tail position reflected the lowest buffeting levels of the nine configurations tested. At the test incidence of $\alpha=20^{\circ}$, breakdown was not observed. In addition, a strong tail vortex formed and interacted with the delta-wing vortex. The computational model differs from the experimental model in the tail support mechanism. In the experiment, the tails were placed in cylindrical tail-support booms aft of the wing. For the computations, the wing and tail were "magnetically suspended," so that no support mechanisms existed.

While the ultimate objective of the delta wing with tails investigation is to understand the unsteady tail-buffeting phenomena, the present study is for a case without vortex breakdown. The computations and the experiment were performed with an angle of attack of $20^{\circ}$ and a Reynolds number of 500,000, for which a laminar boundary layer results. For the experiment, which was performed in an atmospheric tun- 
nel, $M_{\infty}=0.05$ corresponded to the velocity required for the chosen Reynolds number. For the computation, $M_{\infty}=0.3$ because CFL3D is a compressible flow solver, and convergence is slow for very low Mach numbers. In the following sections, the governing equations for the computational method are given and a description of the chimera grid system is provided. A description of the experimental technique is also given. Results for the computational and experimental comparisons are provided in the last section.

\section{Numerical Method}

\section{Governing Equations}

The computational algorithm employed is the three-dimensional Navier-Stokes code CFL3D developed by Thomas et al. ${ }^{13}$ The governing equations, which are the thin-layer approximations to the three-dimensional time-dependent compressible Navier-Stokes equations, can be written in terms of generalized coordinates as

$$
\frac{\partial \widehat{\mathbf{Q}}}{\partial t}+\frac{\partial \widehat{\mathbf{F}}}{\partial \xi}+\frac{\partial \widehat{\mathbf{G}}}{\partial \eta}+\frac{\partial\left(\widehat{\mathbf{H}}-\widehat{\mathbf{H}}_{v}\right)}{\partial \zeta}=0
$$

where

$$
\begin{aligned}
& \widehat{\mathbf{Q}}=\frac{\mathbf{Q}}{J}=\frac{1}{J}\left[\begin{array}{c}
\rho \\
\rho u \\
\rho v \\
\rho w \\
e
\end{array}\right] \\
& \widehat{\mathbf{F}}=\frac{\mathbf{F}}{J}=\frac{1}{J}\left[\begin{array}{c}
\rho U \\
\rho U u+\xi_{x} p \\
\rho U v+\xi_{y} p \\
\rho U w+\xi_{z} p \\
(e+p) U
\end{array}\right] \\
& \widehat{\mathbf{G}}=\frac{\mathbf{G}}{J}=\frac{1}{J}\left[\begin{array}{c}
\rho V \\
\rho V u+\eta_{x} p \\
\rho V v+\eta_{y} p \\
\rho V w+\eta_{z} p \\
(e+p) V
\end{array}\right] \\
& \widehat{\mathbf{H}}=\frac{\mathbf{H}}{J}=\frac{1}{J}\left[\begin{array}{c}
\rho W \\
\rho W u+\zeta_{x} p \\
\rho W v+\zeta_{y} p \\
\rho W w+\zeta_{z} p \\
(e+p) W
\end{array}\right]
\end{aligned}
$$

$$
\begin{aligned}
\widehat{\mathbf{H}}_{v}=\frac{\mathbf{H}_{v}}{J}=\frac{1}{J}\left[\begin{array}{c}
0 \\
\zeta_{x} \tau_{x x}+\zeta_{y} \tau_{x y}+\zeta_{z} \tau_{x z} \\
\zeta_{x} \tau_{x y}+\zeta_{y} \tau_{y y}+\zeta_{z} \tau_{y z} \\
\zeta_{x} \tau_{x z}+\zeta_{y} \tau_{y z}+\zeta_{z} \tau_{z z} \\
\zeta_{x} b_{x}+\zeta_{y} b_{y}+\zeta_{z} b_{z}
\end{array}\right] \\
U=\xi_{x} u+\xi_{y} v+\xi_{z} w \\
V=\eta_{x} u+\eta_{y} v+\eta_{z} w \\
W=\zeta_{x} u+\zeta_{y} v+\zeta_{z} w
\end{aligned}
$$

A general, three-dimensional transformation between the Cartesian variables $(x, y, z)$ and the generalized coordinates $(\xi, \eta, \zeta)$ is implied in equation (1), where $\zeta$ corresponds to the coordinate that is normal to the body surface. The vector $\mathbf{Q}$ represents density, momentum, and total energy per unit volume; $p$ is the pressure defined from the equation of state for an ideal gas:

$$
p=(\gamma-1)\left[e-\frac{\rho}{2}\left(u^{2}+v^{2}+w^{2}\right)\right]
$$

The equations are nondimensionalized in terms of the free-stream density $\widetilde{\rho}_{\infty}$, the free-stream speed of sound $\widetilde{a}_{\infty}$, and the free-stream molecular viscosity coefficient $\tilde{\mu}_{\infty}$. The shear stress and heat flux terms are defined in tensor notation (summation convention implied) as

$$
\begin{gathered}
\tau_{x_{i} x_{j}}=\frac{M_{\infty}}{\operatorname{Re}}\left[\mu\left(\frac{\partial u_{i}}{\partial x_{j}}+\frac{\partial u_{j}}{\partial x_{i}}\right)+\lambda \frac{\partial u_{k}}{\partial x_{k}} \delta_{i j}\right] \\
\dot{q}_{x_{i}}=-\left[\frac{M_{\infty}}{\operatorname{Re} \sigma(\gamma-1)}\right] \frac{\partial a^{2}}{\partial x_{i}} \\
b_{x_{i}}=u_{j} \tau_{x_{i} x_{j}}-\dot{q}_{x_{i}}
\end{gathered}
$$

The chain rule is used to evaluate derivatives with respect to $(x, y, z)$ in terms of $(\xi, \eta, \zeta)$. Consistent with the thin-layer assumption, only those derivatives in the direction normal to the wall $(\zeta)$ are retained in the shear stress and heat flux terms. Equation (1) is closed by the Stokes hypothesis for bulk viscosity $(\lambda+2 \mu / 3=0)$ and Sutherland's law for molecular viscosity. ${ }^{14}$

The CFL3D code also has the capability to solve the Euler equations, which are obtained when the $\widehat{\mathbf{H}}_{v}$ term is omitted from equation (1). Both the Euler and Navier-Stokes equations are utilized for the delta-wing and vertical-tail configuration. The laminar NavierStokes equations are employed for the flow over the wing. Because the main item of interest for this study is the interaction of the wing vortex with the tail and not the tail viscous effects, the Euler equations are used to describe the flow field on the tail grid. 


\section{Grid System}

The delta-wing and vertical-tail configuration is modeled with four grids - a delta-wing grid and three grids that describe the vertical tail. Figure 1 illustrates a typical delta-wing grid. Although the delta wing grid has 65 points in the circumferential direction, 65 points in the radial direction, and 53 points in the longitudinal direction, a coarser grid is shown here for clarity. The grid extends about 1 root chord both upstream and downstream of the wing and approximately 1.5 root chord lengths radially. The cross-sectional grids at the apex and trailing edge are generated algebraically by a sheared Joukowski transformation with clustering near the wing surface to resolve viscous effects. As the cross-sectional grids are interpolated between the wing apex and trailing edge, the minimum spacing increases from about $4.0 \times 10^{-5}$ at the apex to $1.5 \times 10^{-4}$ at the trailing edge.

The vertical tail is modeled with three grids-a main grid and two cap grids. The main tail grid, shown in Fig. 2, was generated with the GRIDGEN grid-generation package. ${ }^{15}$ Composed of 45 stacked O-meshes, the tail grid has 65 points circumferentially and 33 points radially. Because tail viscous effects are not considered here, inviscid spacing is used in the direction normal to the tail. The tail grid system is completed with two cap grids that define the upper and lower surfaces of the tail. The cap grids are each of dimensions $9 \times 11 \times 33$ and maintain the spacing used in the main tail grid. The lower surface cap grid, along with an O-mesh cross section from the main tail grid, is depicted in Fig. 3.

The delta-wing grid and tail grids communicate with one another through interpolation stencils that are obtained with the grid-overlapping (or chimera) scheme developed by Benek et al. ${ }^{9}$ As a simplified example of grid overlapping, consider a cross section of a wing O-mesh that overlaps a Cartesian mesh, as shown in Fig. 4. The wing grid creates a "hole" in the Cartesian grid. Any cell center point of the Cartesian grid located within this hole is designated a "hole point." The first two "nonhole" cell center points of the Cartesian grid that border a hole point both vertically and horizontally are labelled "fringe points." In the figure, only the upper half of the wing grid is drawn to provide a better view of the hole and its fringe points. Each fringe point of the Cartesian grid falls within a "target cell" of the wing grid.

A searching algorithm is used to identify the particular eight points that surround the fringe point, which form the hexahedral target cell. The search begins with an initial guess for the target cell. Next, the current target cell is isoparametrically mapped into a unit cube in computational space. The same mapping is then applied to the fringe point; if the mapped fringe point lies in the same unit cube as the current target cell, then that target cell in fact surrounds the fringe point. If the mapped fringe point lies outside the unit cube, then the current target cell is not the correct choice. However, the magnitude and direction of the mapped fringe point relative to the current target cell may be used to choose a new guess for the target cell. The mapping process is repeated until the correct target cell is identified. With the correct target cell identified, the data are transferred from the target cell to the fringe point with trilinear interpolation in computational space. Outer boundary values of the wing grid are determined in a similar manner. The MultiGeometry Grid Embedder (MaGGiE) code, written specifically for CFL3D by Baysal et al., ${ }^{16}$ was used to determine interpolation information between the grids. However, some modifications have been made to the original version of MaGGiE. For example, rather than use a single layer of fringe points for the interpolations across grids as in the original version of MaGGiE, two layers are now utilized for greater accuracy. The interpolation and boundary stencils obtained from MaGGiE provide the information necessary for communication across the grids within the flow solver.

For the delta-wing and tail configuration, the tail grid creates a hole in the delta-wing grid in a manner similar to the wing O-mesh and Cartesian grid example, and interpolation stencils between the grids are determined with MaGGiE. Thus, the complete grid system is composed of a tail grid, two tail cap grids, and a delta-wing grid with a hole cut away in the region of the tail. A partial view of this hole is shown in Fig. 5. The total number of grid points for the configuration is 326,984 , although any points located within the hole are omitted from the calculation.

\section{Boundary Conditions}

For the delta-wing surface, a no-slip boundary condition is applied with the velocity set to zero, the temperature set to free-stream total temperature, and the pressure determined from a zero normal pressuregradient assumption. Symmetry conditions are applied along the vertical plane of symmetry. A quasi-onedimensional characteristic analysis is used along the far-field longitudinal and circumferential boundaries with the assumption that free-stream conditions exist exterior to the boundary. 
For the vertical-tail main grid and cap grids, flow tangency is enforced at the body surface. In addition, the density and pressure boundary points are set equal to the first cell center values of density and pressure, respectively. Chimera grid interpolations supply all tail grid outer boundaries with flow-field information from the delta-wing grid.

\section{Experimental Method}

The experimental investigation was conducted in the NASA Langley Research Center Basic Aerodynamic Research Tunnel. The tunnel is an open-return wind tunnel with a closed rectangular test section that is 28 in. $(0.71 \mathrm{~m})$ high, $40 \mathrm{in}$. $(1.02 \mathrm{~m})$ wide, and $10 \mathrm{ft}(3.05 \mathrm{~m})$ long. The maximum flow velocity in the test section is $220 \mathrm{ft} / \mathrm{sec}$, which yields a $R e / \mathrm{ft}$ of 1.4 million. The air that enters the test section is conditioned by a honeycomb, four antiturbulence screens, and a contraction ratio of 11:1. The flow conditioners enable a low-turbulence, uniform flow with turbulence intensities in the test section that range from 0.03 percent to 0.09 percent for dynamic pressures of $3 \mathrm{lb} / \mathrm{ft}^{2}$ to $30 \mathrm{lb} / \mathrm{ft}^{2}\left(50 \leq|\widetilde{\mathbf{V}}|_{\infty} \leq 160 \mathrm{ft} / \mathrm{sec}\right)$.

The $76^{\circ}(A R=1)$ delta-wing model is shown in Fig. 6. It has a nominally sharp leading edge, a flat upper surface, and a triangular cross section. The root chord is $1.5 \mathrm{ft}(0.46 \mathrm{~m})$ and the maximum thickness is 2.1 percent of the root chord at $\widetilde{x} / \widetilde{L}=0.9$. The leading-edge radius is $0.005 \mathrm{in}$. $(0.13 \mathrm{~mm})$, and roughness on the upper and lower surfaces is $32 \mu \mathrm{in}$ $(0.8 \mu \mathrm{m})$ and $64 \mu \mathrm{in}(1.6 \mu \mathrm{m})$, respectively.

The planform was supported by a short sting mounted on a post support. The sting was attached to the underside of the model to reduce mounting interference on the flat upper surface of the delta wing. The post-mount height was fixed for the investigation. Consequently, the model moved off the tunnel centerline as the angle of attack changed.

Two measurement techniques were used for comparison with the computational results. Initially, a laser light sheet was used to visualize the flow field at a series of chordwise stations. A light sheet normal to the upper surface of the delta wing was produced by a 6watt argon ion beam and a cylindrical lens. The smoke, which was vaporized propylene glycol, was introduced upstream of the flow conditioners at the inlet.

The light-sheet flow visualizations were used to identify global features of the flow field, to map the vortex trajectories, and to determine the location of vortex breakdown. To map the vortex trajectories, video frames were digitally processed and displayed with flow analysis software in three-dimensional space. ${ }^{17}$

To complement the flow visualization, flow-field velocities and pressures were measured at the chordwise stations $\tilde{x} / \widetilde{L}=1.4$ and 1.6. These data were acquired with a five-hole probe mounted in a five-degreeof-freedom traverse. The nonuniform experimental grid had a spacing of $\widetilde{y} / \widetilde{s}=0.03$ on the port side of the model and a starboard spacing of $\widetilde{y} / \widetilde{s}=0.015$. The grid was uniformly spaced with $\tilde{z} / \widetilde{s}=0.015$ in the direction normal to the wing upper surface.

The five-hole probe can measure total flow angles $\theta$ (with respect to the probe axis) up to $50^{\circ}$ with an associated error of $\pm 1.0^{\circ}$ for $\theta<40^{\circ}$ and $\pm 2.0^{\circ}$ for $40^{\circ}<\theta<50^{\circ}$. The flow at these stations, however, contained flow angles that exceeded $50^{\circ}$. Hence, the data were acquired with the probe set at two different angles. Initially, the probe angle was set at $0^{\circ}$ relative to the free-stream flow. The survey plane was then divided into regions based on the total flow angle relative to the probe. Regions where the flow angle exceeded $50^{\circ}$ were measured again with the probe inclined at $-15^{\circ}$. The combined data set contained no flow angles greater than $49^{\circ}$.

\section{Results}

The flow field over a highly swept delta wing at high angles of attack is characterized by the counterrotating vortices that are generated at the sharp leading edges of the wing. Figure 7 illustrates this flow field with the nondimensional computational stagnation pressure $\left(\widetilde{p}_{0} / \widetilde{p}_{\infty}\right)$ contours plotted at several constant $\widetilde{x} / \widetilde{L}$ stations on the delta wing with vertical tails. The calculation was performed with an angle of attack of $20^{\circ}$ and a Reynolds number of 500,000. Although the flow field was calculated for only one side of the wing (symmetry was assumed and interactions were ignored), the results have been reflected in the figure to show both primary vortices. Also visible in the figure are the wing secondary vortices.

A front view of the computational configuration is presented in Fig. 8. Again, the solution is reflected to show both sides of the wing and both tails. Yellow particle traces, which are released near the apex of the delta wing, track the paths of the wing primary and secondary vortices. Red particle traces reveal two vortices generated at the sharp edges of the tail. Figure 9 provides a close-up of the tail region for a better view of the flow features. Nondimensional computational helicity $\left(\widetilde{\boldsymbol{\Omega}} \widetilde{L} / \widetilde{a}_{\infty} \cdot \widetilde{\mathbf{V}} / \widetilde{a}_{\infty}\right)$ contours have been added 
to illustrate the sizes, shapes, and relative rotational directions of the wing and tail vortices. The color bars in the figures provide a key for the helicity contour levels; the particle traces are colored only to distinguish between the vortices originating at the wing (yellow) and the tail vortices (red). In Fig. 9(a), the helicity contours are plotted at the wing trailing edge $(\widetilde{x} / \widetilde{L}=1.0)$. The helicity contours exhibit the opposing rotational directions of the wing primary and secondary vortices. Yellow particle traces pass through the wing primary and secondary vortex cores. Figure 9(b) illustrates the helicity contours at $\widetilde{x} / \widetilde{L}=1.3$. At this cross section, the wing primary vortex still appears quite strong, and the secondary vortex is beginning to diffuse. The helicity contours also reveal the two vortices released from the sharp leading edges of the tail. Red particle traces released along the front edge of the tail pass through the tail leading-edge vortex. Spanwise flow gives the tail an effective angle of attack; the vortex is generated at the highly swept, sharp leading edge of the tail in the same manner that the wing primary vortex forms because of the wing sharp leading edge. The helicity contours indicate that the tail leading-edge vortex rotates in the opposite direction of the wing primary vortex. Red particle traces released near the sharp edge on the lower, outboard side of the tail pass through the second vortex, which rotates in the same direction as the wing primary vortex. In Fig. 9(c), the helicity contours are plotted farther downstream at $\widetilde{x} / \widetilde{L}=1.5$. While the two tail vortices still maintain their shapes at this cross section, the wing primary vortex has practically disappeared.

Side-by-side comparisons of the computational and experimental flow fields are shown in Fig. 10. On the left side of the figures, contour levels represent stagnation pressure values obtained computationally; on the right side of the figures, experimental smoke flow visualizations, which are artificially colored to reflect the vortex shape and position, are depicted. These flow visualizations were obtained with a recently developed technique for transferring experimental flow visualizations into the same three-dimensional environment with the computational data. ${ }^{17}$ The yellow and red lines track the experimental wing primary-vortex core and the tail leading-edge vortex core, respectively. These core paths were obtained with the method developed by Stacy et al. ${ }^{17}$ The experimental core trajectories have also been plotted on the computational side of the figures to provide a frame of reference for the computational core paths. In Fig. 10(a), the computational stagnation pressure contours and experimental flow visualizations are shown at the trailing edge of the delta wing. Good agreement is noted for the size and shape of the wing primary and secondary vortices. Figure 10(b) shows a similar view farther downstream at $\widetilde{x} / \widetilde{L}=1.2$. Again, the size and shape of the wing primary vortices and the vortex-core locations are in good agreement for the computation and the experiment. At this station, the wing secondary vortex is more pronounced on the computational side. In Fig. 10 (c), the computational stagnation pressure contours and the experimental smoke densities are illustrated at $\widetilde{x} / \widetilde{L}=1.4$. The tail leading-edge vortices, which have become evident at this cross section, begin to push the wing primary vortex inboard, which is shown by both computation and experiment. On the computational side, the vortex generated at the sharp edge on the lower, outboard side of the tail is also visible. No corresponding vortex is visible on the experimental side because of the cylindrical tail boom, which conceals the sharp edge of the tails. In the computations, the wing and tail are magnetically suspended and thus have no support beam that connects the tail to the wing as in the experiment. Figure 10(d) shows the results at $\widetilde{x} / \widetilde{L}=1.5$. The computations indicate less rotation of the wing primary and tail leading-edge vortices and a more elongated core region for the primary vortex.

In Fig. 11, the computational and experimental wing primary and tail leading-edge vortex-core trajectories are compared for both the wing alone and the wing with vertical tails. Figure 11(a) provides an overhead view of the wing and tail; Fig. 11(b) gives a view from the side. Both views show close agreement between the computational and experimental results over the wing. In the region of the tail, both the computational and experimental results indicate an upward and outboard movement of the wing primary-vortex core caused by the presence of the tail. A good correlation is also seen for the tail leading-edge vortex-core path.

As indicated previously in Figs. 9 and 10, a loss of strength in the wing primary vortex is observed computationally as the vortex progresses downstream of the delta wing. Likewise, in Fig. 12, a comparison of computational (left) and experimental (right) nondimensional axial vorticity $\left(\widetilde{\Omega}_{\mathrm{x}} \widetilde{L} /|\widetilde{\mathbf{V}}|_{\infty}\right)$ levels at $\tilde{x} / \widetilde{L}=1.4$ reveals a deficiency in the computational vorticity magnitudes in the wing primary-vortex-core region. This growing weakness of the computational wing primary vortex becomes even more evident in comparisons with experimental vorticity contours at $\widetilde{x} / \widetilde{L}=1.6$ (not shown here). Based on previous research, ${ }^{8}$ this loss of strength can be attributed to a lack of sufficient grid resolution in the region where the vortex is located. Figure 13 shows the improved 
core strength predictions obtained with 2 levels of grid embedding.

\section{Conclusions}

Computations have been performed, and an initial experimental data base has been obtained for a deltawing and vertical-tail configuration in a joint effort to understand the physics of the vortex and tail interaction process that is encountered in modern fighter aircraft. Comparisons have been presented for a laminar case with an angle of attack of $20^{\circ}$ and a Reynolds number of 500,000. Vortex sizes and shapes from the computations, as well as vortex-core trajectories, compare favorably with the experimental results. Some differences in the wing primary-vortex shape and strength are observed as the vortices pass over the far end of the tail. Grid embedding improves the quality of the solution in this region. Also, an additional vortex is generated at the tail because of the absence of a tail support mechanism in the computation. Future studies will continue to investigate the unsteady phenomena that occur at higher angles of attack.

\section{Acknowledgments}

Sincere appreciation is extended to Dr. R. T. Biedron and Dr. J. L. Thomas for their efforts and advice in regard to the computational portion of the investigation.

This research was supported (in part) by the National Aeronautics and Space Administration under contract no. NAS1-19672.

The experimental portion of the research was performed in conjunction with McDonnell Douglas Government Aerospace-East (MDGA) under a cooperative Memorandum of Agreement. The effort at MDGA was headed by Jack Jacobs and Marty Ferman.

\section{References}

[1] Wentz, Jr., W. H., "Vortex-Fin Interaction on a Fighter Aircraft," AIAA 87-2474-CP, 1987.

[2] Lee, B. H. K., and Brown, D., "Wind-Tunnel Studies of F/A-18 Tail Buffet," Journal of Aircraft, Vol. 24, No. 1, 1992.

[3] Martin, C. A., and Thompson, D. H., "Scale Model Measurements of Fin Buffet Due to Vortex Bursting on F/A-18," AGARD CP-497, Paper No. 12, 1992.
[4] Rizk, Y., "Numerical Prediction of the Unsteady Flowfield Around the F-18 Aircraft at Large Incidence," AIAA 91-0020, 1991.

[5] Rizk, Y., and Guruswamy, G. P., "Numerical Investigation of Tail Buffet on F-18 Aircraft," AIAA 92-2673, 1992.

[6] Washburn, A. E., Jenkins, L. N., and Ferman, M. A., "Experimental Investigation of Vortex-Fin Interaction," AIAA 93-0050, 1993.

[7] Taylor, S. L., Kjelgaard, S. O., Weston, R. P., Thomas, J. L., and Sellers, III., W. L., "Experimental and Computational Study of the Subsonic Flow About a $75^{\circ}$ Swept Delta Wing," AIAA 872425, 1987.

[8] Krist, S. L., Thomas, J. L., Sellers, III., W. L., and Kjelgaard, S. O., "An Embedded Grid Formulation Applied to a Delta Wing," AIAA 90-0429, 1990.

[9] Benek, J. A., Steger, J. L., Dougherty, F. C., and Buning, P. G., "Chimera: A Grid-Embedding Technique," AEDC-TR-85-64, 1986.

[10] Donegan, T. L., Benek, J. A., and Erickson, Jr., J. C., "Calculation of Transonic Wall Interference," AIAA 87-1432, 1987.

[11] Rizk, Y., Schiff, L., and Gee, K., "Numerical Simulation of the Viscous Flow Around the F-18 at High Angles of Attack," AIAA 90-2999, 1990.

[12] Buning, P. G., Chiu, I. T., Obayashi, S., Rizk, Y. M., and Steger, J. L., "Numerical Simulation of the Integrated Space Shuttle Vehicle in Ascent," AIAA 88-4359CP, 1988.

[13] Thomas, J. L., Krist, S. L., and Anderson, W. K., "Navier-Stokes Computations of Vortical Flows Over Low-Aspect-Ratio Wings," AIAA Journal, Vol. 28, No. 2, pp. 205-212, 1990.

[14] White, F. M., Viscous Fluid Flow. McGraw-Hill Book Company, 1974.

[15] Steinbrenner, J. P., Chawner, J. R., and Fouts, C. L., "The GRIDGEN 3D Multiple Block Grid Generation System," WRDC-TR-90-3022, 1990.

[16] Baysal, O., Fouladi, K., and Lessard, V. R., "A Multigrid and Upwind Viscous Flow Solver on 3-D Overlapped and Embedded Grids," AIAA Journal, Vol. 29, No. 6, pp. 903-910, 1990.

[17] Stacy, K., Severance, K., and Childers, B. A., "Computer-Aided Light Sheet Flow Visualization," AIAA 93-2915-CP, 1993. 


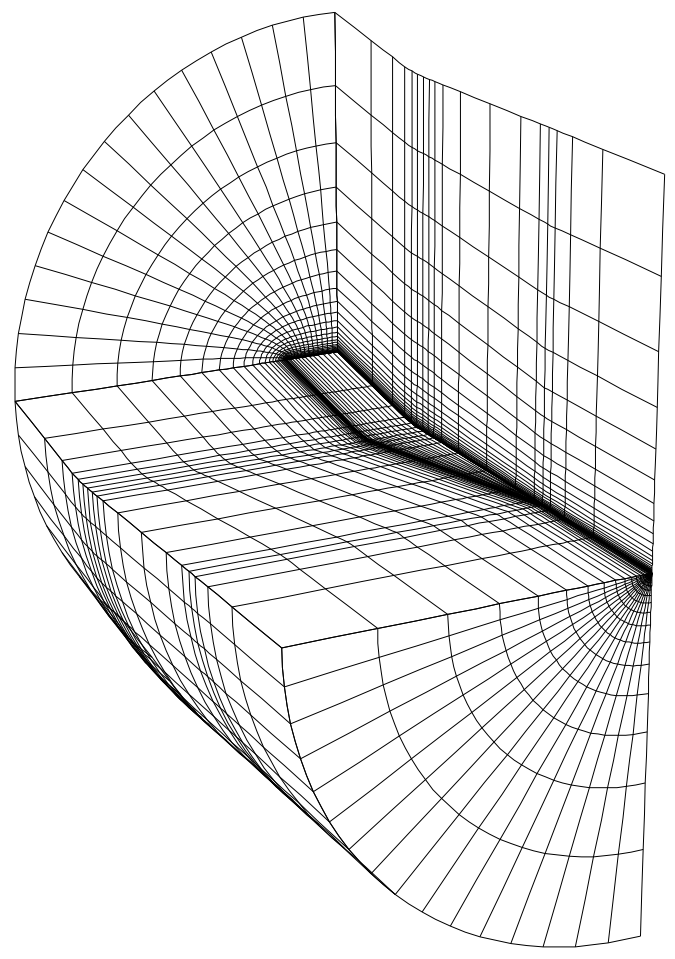

Figure 1. Three-dimensional delta-wing grid $(33 \times 33 \times 19)$.

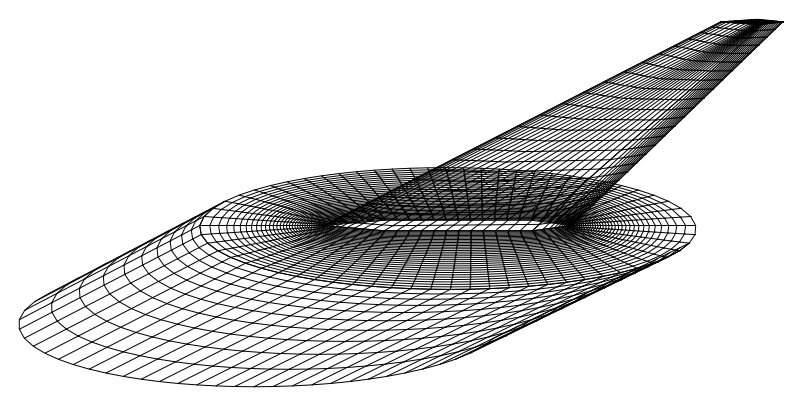

Figure 2. Vertical tail surface grid and lower portion of tail O-mesh $(65 \times 33 \times 45)$.

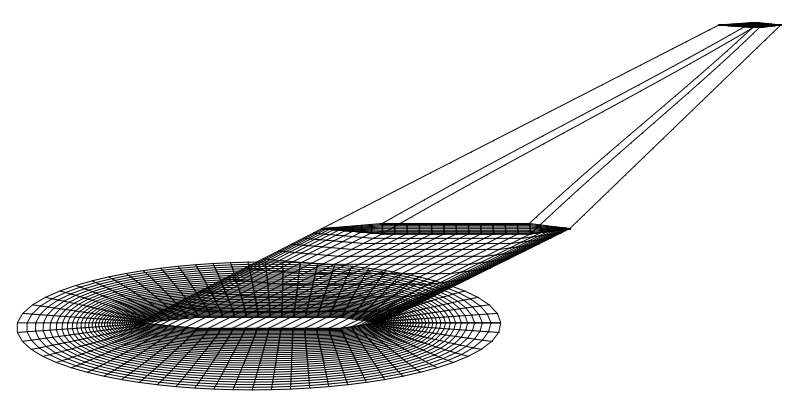

Figure 3. Tail cap grid $(9 \times 11 \times 33)$ below vertical tail and vertical-tail cross-sectional O-mesh.
Target cell

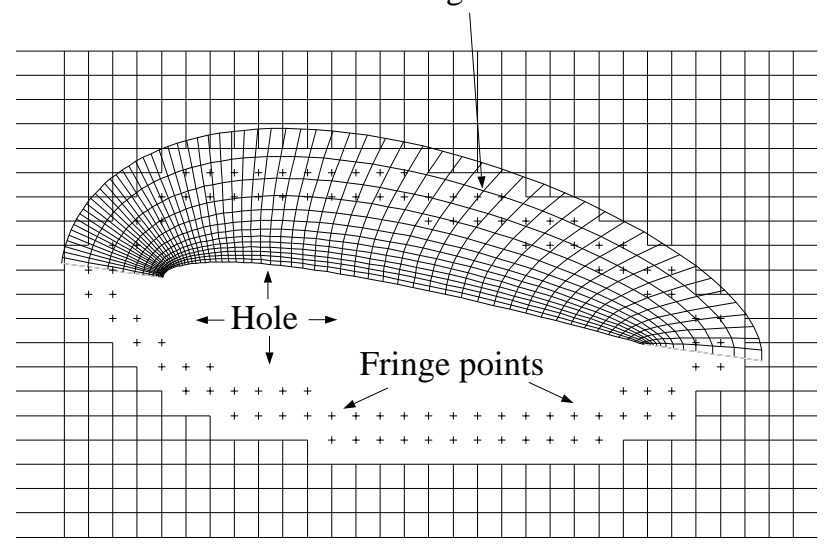

Figure 4. Hole carved out of Cartesian mesh for wing O-mesh.

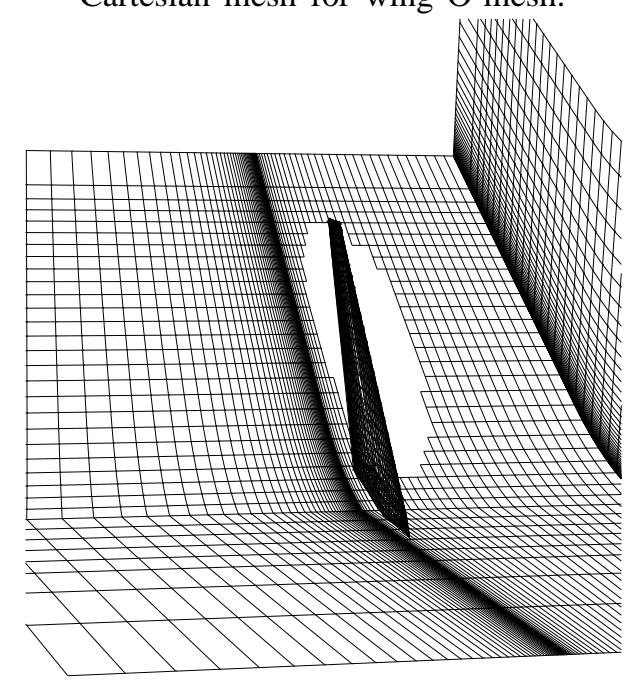

Figure 5. Close-up of portion of hole cut away in delta-wing mesh for vertical-tail grid.
Figure 6. Experimental model of $A R=1$ delta wing with vertical tails. 
(a) $\tilde{x} / \widetilde{L}=1.0$

Figure 7. Computational stagnation pressure contours.

(b) $\widetilde{x} / \widetilde{L}=1.3$

Figure 8. Computational particle traces.

(c) $\tilde{x} / \tilde{L}=1.5$

Figure 9. Computational helicity contours. 
(a) $\tilde{x} / \widetilde{L}=1.0$

(b) $\tilde{x} / \widetilde{L}=1.2$

(c) $\tilde{x} / \widetilde{L}=1.4$

(d) $\tilde{x} / \widetilde{L}=1.5$

Figure 10. Computational stagnation pressure contours (left side of figures) and experimental smoke visualization images (right side of figures).

(a) Overhead view.

Figure 11. Computational and experimental vortex-core trajectories. (Continued ....) 
(b) Side view.

Figure 11. Computational and experimental vortex-core trajectories.

Figure 12. Computational (left) and experimental (right) axial vorticity contours, $\widetilde{x} / \widetilde{L}=1.4$.

Figure 13. Computational with grid embedding (left) and experimental (right) axial vorticity contours, $\widetilde{x} / \widetilde{L}=1.4$. 